Check for updates

Cite this: Phys. Chem. Chem. Phys., 2019, 21, 4063

Received 9th November 2018, Accepted 22nd January 2019

DOI: $10.1039 / c 8 c p 06961 a$

rsc.li/pccp

\section{A tool box to ascertain the nature of doping and photoresponse in single-walled carbon nanotubes $\dagger$}

\author{
Ana Santidrián, (D)*ab José M. González-Dominguez, (D) bc Valentin Diez-Cabanes, ${ }^{d}$ \\ Javier Hernández-Ferrer, $\mathbb{D}^{\mathrm{b}}$ Wolfgang K. Maser, (iD) ${ }^{\mathrm{b}}$ Ana M. Benito, (iD ${ }^{\mathrm{b}}$ \\ Alejandro Anśon-Casaos, ${ }^{b}$ Jérôme Cornil, (iD d Tatiana Da Ros $^{d}$ and Martin Kalbáč (D) *a
}

\begin{abstract}
The effect of doping on the electronic properties in bulk single-walled carbon nanotube (SWCNT) samples is studied for the first time using a new in situ Raman spectroelectrochemical method, and further verified by DFT calculations and photoresponse. We use p-/n-doped SWCNTs prepared by diazonium reactions as a versatile chemical strategy to control the SWCNT behavior. The measured and calculated data testify an acceptor effect of 4-aminobenzenesulfonic acid (p-doping), and a donor effect ( $n$-doping) in the case of benzyl alcohol. In addition, pristine and covalently functionalized SWCNTs were used for the preparation of photoactive film electrodes. The photocathodic current in the photoelectrochemical cell is consistently modulated by the doping group. These results validate the in situ Raman spectroelectrochemistry as a unique tool box for predicting the electronic properties of functionalized SWCNTs in the form of thin films and their operational functionality in thin film devices for future optoelectronic applications.
\end{abstract}

\section{Introduction}

In the last few decades, unique carbon allotropes have emerged based on $\mathrm{sp}^{2}$ bonds, including fullerenes, graphene and carbon nanotubes (CNTs). ${ }^{1}$ Single-walled carbon nanotubes (SWCNTs) are intriguing from the chemical point of view owing to their size and orbital structure and the existence of a broad range of chiralities. Progressively, SWCNTs are finding their way into critical electronic applications such as sensors or energy devices, which are becoming a commercial reality. ${ }^{2-5}$

To fully exploit the potential of SWCNTs, physicochemical methods have been developed to process pristine SWCNTs and adjust their optoelectronic properties. Among them, covalent

\footnotetext{
${ }^{a} J$. Heyrovský Institute of Physical Chemistry, Academy of Sciences of the Czech Republic, Dolejskova 3, 18223 Prague 8, Czech Republic.

E-mail: martin.kalbac@jh-inst.cas.cz

${ }^{b}$ Instituto de Carboquimica ICB-CSIC, Miguel Luesma Castan 4, 50018 Zaragoza, Spain.E-mail:asantidrian@icb.csic.es

${ }^{c}$ INSTM Unit of Trieste, Department of Chemical and Pharmaceutical Sciences, University of Trieste, Via Licio Giorgieri 1, 34127 Trieste, Italy

${ }^{d}$ University of Mons, Place du Parc 20, 7000 Mons, Belgium

$\dagger$ Electronic supplementary information (ESI) available: Further details of the purification of SWCNTs, reagents and solvents used; deconvolution of the D-band and the G-band; DOS of the SWCNTs for the structure optimized with SIESTA and CRYSTAL; UV-vis absorbance spectra of SWCNTs, A-SWCNTs and B-SWCNTs; the RBM region of Raman spectra at different $E_{\text {app }}$ values for A-SWCNTs and B-SWCNTs; dark voltammograms for SWCNTs, A-SWCNTs and B-SWCNTs. See DOI: $10.1039 / \mathrm{c} 8 \mathrm{cp} 06961 \mathrm{a}$
}

functionalization is one of the most efficient strategies. In this way, SWCNTs have shown enough chemical versatility to undergo reactions not only with electron-deficient species, such as radicals, carbene or nitrene compounds, but also with electron-rich reagents such as alkali metals. ${ }^{6-8}$ Most of the chemical functionalization procedures are sensitive to the electronic properties of the SWCNTs and can also lead to significant changes in their electronic structure. Previous works have discussed how SWCNTs can be selectively doped through functionalization, in which electrons or positive holes are introduced into their structure by interaction with either electron donor or acceptor groups. ${ }^{9-12}$

If there were one particular kind of covalent chemistry applied to SWCNTs as the most trusted methodology to achieve chemical doping, it would undoubtedly be the diazoniumbased reactions. ${ }^{9,13}$ Briefly, these consist of the thermally or electrochemically induced reaction of a diazo compound that evolves into an aryl radical capable of creating a cascade of radical reactions between SWCNTs and aryl moieties, ending up in stable $\mathrm{C}-\mathrm{C}$ bonds between both units. ${ }^{14}$ This scheme has been widely employed not only for altering the SWCNT electronic structure but also for grafting functional moieties with multiple anchoring possibilities. ${ }^{15}$ More recently, the diazonium chemistry of SWCNTs has been used for the creation of bright, fluorescent quantum defects. Changes in SWCNT photoluminescence through covalent sidewall functionalization have proven enormously versatile. Some studies have reported that several photoluminescence features (such as emission energy and maximum brightness) 
can be chemically tuned using withdrawing/donating substituents on the aryl functional group. ${ }^{16,17}$ It has even been shown that SWCNTs can be photoexcited to induce an acceleration in diazonium functionalization. ${ }^{18}$

Even if the intended alteration of the SWCNTs' electronic structure is a fact, by means of diazonium chemistry or any other approach, on many occasions there is a need to unravel the nature of doping (negative, $\mathrm{n}$ - or positive, $\mathrm{p}$-) and its extension. Transport measurements ${ }^{19,20}$ or thermoelectric power measurements, ${ }^{21,22}$ that require a sophisticated system, can be performed to investigate the Fermi level changes. However, these techniques are not usually adapted to distinguish between n- or p-doping. Optical techniques, such as near-infrared fluorescence and photoluminescence excitation, albeit very powerful, are exclusively limited to individual SWCNTs, which is often unviable in many samples and procedures. ${ }^{23,24}$

Raman spectroscopy is much more accessible and powerful as it is a non-destructive, contactless and quick technique that requires relatively simple or no preparation, and is greatly sensitive to changes in the physical and chemical properties of SWCNTs. ${ }^{25}$ To characterize doping, Raman spectroscopy primarily relies on G-band shifts. ${ }^{26}$ However, these changes in the G-band are sometimes masked by other effects, such as strain. In addition, the G-band frequency is only weakly dependent on the tubes' diameter, and therefore, in nanotube bundles one cannot extract shifts for individual tubes. Despite a few successful attempts reported in the literature, ${ }^{27-29}$ an assignment of the up/down shift to a given doping process is still not straightforward. Therefore, alternative methods to determine the qualitative differentiation between $\mathrm{n}$ - and $\mathrm{p}$-doping induced by functionalization would be beneficial. Ideally, the method should be suitable to tailor the electronic effects upon functionalization by various functional groups. A prospective method to address this task is in situ Raman spectroelectrochemistry, as it allows changes in the Raman spectra of SWCNTs during doping to be followed in a wide potential range in a precise and reproducible way. ${ }^{30-33}$

In the present work, we refer to a previously described functionalization scheme, based on diazonium chemistry. We controlled the experimental variables to end up in a high level of sidewall decoration with one or several functional groups, which can be further derivatized, and minimizing the oligomerization of reactive species. ${ }^{34}$ This approach was adapted to obtain functionalized SWCNTs with two different grafted moieties, achieving the dual effect of high functionalization level and the tuning of their electronic structure. In situ Raman spectroelectrochemistry is used to investigate the radial breathing mode (RBM) and to assess the electronic structure of covalently functionalized SWCNT bundles. The proposed methodology allows unambiguous distinguishing between chemical $\mathrm{p}$ - and n-doping. The approach has been tested in bulk samples of SWCNTs, which represent the majority of applicable cases. The experimental results were supported by density functional theory (DFT) calculations. Furthermore, changes in the photoresponse of SWCNT films were recorded, as a proof of principle, confirming the effect of $n$-/p-doping when a specific functional group is attached to the SWCNTs.

\section{Experimental}

\section{Materials and reagents}

Pristine SWCNTs produced by the HiPco ${ }^{\circledR}$ process were purchased from Unidym ${ }^{\mathrm{TM}}$. The raw material was purified in-house using a non-oxidative method. ${ }^{34}$ All other reagents and solvents were purchased from commercial sources and used without further treatment (details in the $\mathrm{ESI} \dagger$ ).

\section{Functionalization of SWCNTs}

Functionalization by diazonium-based radical addition starts with the reaction of a suitable aniline precursor with the organic nitrite. Briefly, purified SWCNTs were dispersed in $N, N$-dimethylformamide (DMF), aided by ultrasonication (5-10 $\mathrm{min}$ ) at a concentration of about $1 \mathrm{mg} \mathrm{mL}{ }^{-1}$. Then, 1.2 equiv. (per mol of $\mathrm{C}$ ) of the desired aniline were incorporated (A-SWCNTs were functionalized using 4-aminobenzenesulfonic acid and B-SWCNTs using 4-aminobenzyl alcohol), and the system was stabilized at $80{ }^{\circ} \mathrm{C}$. Afterwards, 3.2 equiv. of isopentyl nitrite per equivalent of aniline were dropwise incorporated into the system, and the mixture was allowed to react at $80{ }^{\circ} \mathrm{C}$ for $1 \mathrm{~h}$. The whole reaction medium was then filtered (using a hydrophilic Teflon membrane pore size of $0.1 \mu \mathrm{m}$, Omnipore ${ }^{\circledR}$ ) and rinsed with DMF until the filtrate was colourless. The functionalized SWCNTs (A-SWCNTs and B-SWCNTs) were alternatively redispersed and filtered in DMF, water and methanol at $1 \mathrm{mg} \mathrm{mL}^{-1}$ several times. Finally, the filtered solid sample was rinsed in the filter with diethyl ether and left to dry at room temperature under vacuum.

\section{Characterization techniques}

Thermogravimetric analysis (TGA) was done using a TGA Q500 instrument (TA instruments) under a flow of $\mathrm{N}_{2}\left(90 \mathrm{~mL} \mathrm{~min}{ }^{-1}\right)$ following a temperature programme that consisted of the isothermal equilibration of the sample at $100{ }^{\circ} \mathrm{C}$ for $20 \mathrm{~min}$, followed by a ramp of $10{ }^{\circ} \mathrm{C} \min ^{-1}$ to $800{ }^{\circ} \mathrm{C}$. The samples ranged from 1 to $2 \mathrm{mg}$.

The ultraviolet/visible (UV/vis) spectroscopic measurements were done using a Shimadzu UV-2401PC spectrophotometer in quartz cuvettes with a path length of $1 \mathrm{~cm}$.

\section{Raman spectroscopy and in situ Raman spectroelectrochemistry}

Raman spectra were acquired using a LabRAM HR Raman spectrometer (Horiba Jobin-Yvon), provided with laser excitation energies of $2.33 \mathrm{eV}$ (532 nm, Ar/Kr laser, Coherent) and $1.96 \mathrm{eV}$ $(633 \mathrm{~nm}, \mathrm{He}-\mathrm{Ne})$. A $50 \times$ objective was used with a laser spot of about $1 \mu \mathrm{m}$. The laser power was around $1 \mathrm{~mW}$ and the spectral resolution was $1 \mathrm{~cm}^{-1}$. For the determination of positions and intensity ratios, Raman spectra in the tangential region (the G-band) were fitted by one Breit-Wigner-Fano (BWF) line shape and two Lorentzian functions (L1 and L2), which were assigned to the LO mode of metallic tubes (BWF), the TO mode of semiconducting tubes (L1) and the combination of the TO mode of metallic tubes and the LO mode of semiconducting tubes (L2). The D-band was fitted by a Lorentzian line shape (see Fig. S1 in the ESI $\dagger$ ). 
The three-electrode electrochemical cell for in situ Raman spectroelectrochemistry was assembled in a glove box. The working electrode was prepared by drop casting SWCNTs on a Pt wire undergoing a sonicated dispersion of SWCNTs in methanol. Another Pt wire was used as the counter electrode and a silver $(\mathrm{Ag})$ wire as the reference electrode. Calibration for the $\mathrm{Ag}$ pseudoreference electrode was performed via cyclic voltammetry $(\mathrm{CV})$ and the ferrocenium/ferrocene $\left(\mathrm{Fc}^{+} / \mathrm{Fc}\right)$ redox couple was used as an internal standard because of its ideal reversible behaviour, being the potential of the $\mathrm{Fc}^{+} / \mathrm{Fc}$ couple versus an Ag electrode:

$$
E_{\mathrm{Fc} / \mathrm{Fc}^{+}(\mathrm{Pt})}-E_{\mathrm{Ag}}=540 \mathrm{mV}
$$

Subsequently, ${ }^{35}$ the potential of the Ag pseudoreference electrode is calculated to be $-126 \mathrm{mV}$ vs. the $\mathrm{Ag} / \mathrm{AgCl}(3 \mathrm{M} \mathrm{KCl})$ reference electrode: ${ }^{35}$

$$
E_{\mathrm{Ag}}-E_{\mathrm{Ag} / \mathrm{AgCl}}=-126 \mathrm{mV}
$$

The electrolyte solution was $0.2 \mathrm{M} \mathrm{LiClO}_{4}$ (Sigma Aldrich) dissolved in dry acetonitrile. An Autolab PGSTAT (Ecochemie) potentiostat was used to apply potential. The potential was ramped to $\pm 1.5 \mathrm{~V}$ in steps of $0.1 \mathrm{~V}$, and Raman spectra were acquired at a constant potential at every step.

\section{Theoretical modelling}

The geometry of the unit cells has been optimized at the DFT level $^{36}$ using the SIESTA 3.2 package $^{37}$ within the Generalized Gradient Approximation (GGA) with the Perdew-Burke-Ernzerhof (PBE) exchange-correlation functional ${ }^{38}$ and periodic boundary conditions. The valence electrons are described within the Linear Combination of Atomic Orbitals (LCAO) approximation using a Double Zeta + Polarization (DZP) basis set $^{39}$ whereas TroullierMartins pseudopotentials ${ }^{40}$ are used for the description of the core electrons. We use a mesh cut-off of 250 Ry and a $k$-sampling of 6 $(4 \vec{k})$ for the SWCNT $(9,0)$ and $12(7 \vec{k})$ for the $\operatorname{SWCNT}(15,0)$ in the Monkhorst-Pack scheme, where the numbers in parentheses represent the $k$-points in the first Brillouin zone.

The electronic properties have been calculated by performing DFT calculations using the CRYSTAL09 package ${ }^{41}$ on the previously optimized structures, using the PBE hybrid functional $(\mathrm{PBE} 0)^{42}$ and a 6-21G* ${ }^{*}$ basis set; it should be noted that there are no major differences in the studied properties when optimizing the structures with the CRYSTAL program at the PBE0 level (see Fig. S2, ESI $\dagger$ ); it should also be noted that SIESTA has demonstrated its ability to provide reliable optimized structures for large systems, such as the functionalized SWCNTs studied in this work, in a reasonable computational time; however, it is not able to calculate 1-D periodic properties and use hybrid functionals, thus explaining the choice of CRYSTAL. According to previous works, the PBE0 hybrid functional proved to describe the electronic properties in SWCNTs properly. ${ }^{43}$ The threshold on the self-consistent field (SCF) method energy was set to $10^{-7} \mathrm{Ha}$. The level of accuracy when evaluating the Coulomb and Hartree-Fock exchange integrals is controlled by tolerance values of 888816 . The $k$-sampling in the MonkhorstPack scheme used for calculating the electronic properties was

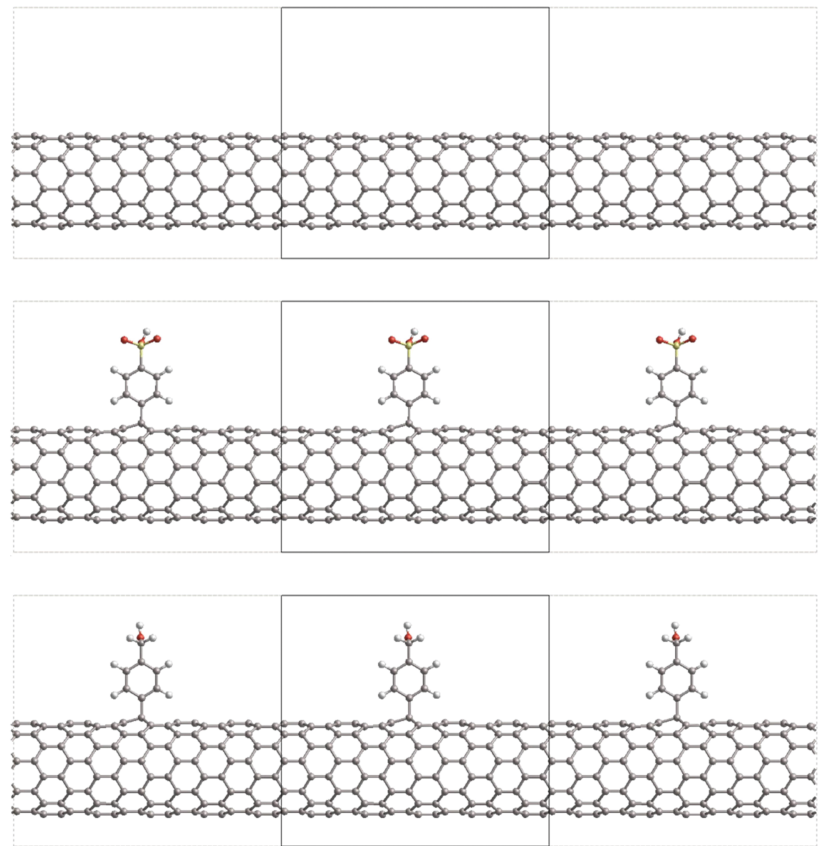

Fig. 1 View of the unit cell of SWCNTs (top), A-SWCNTs (middle) and B-SWCNTs (bottom) for the $(9,0)$ SWCNT family.

the same as for the optimization step. According to the experimental data (as discussed later in the Results section), the ratio of carbon atoms functionalized vs. the total number of carbon atoms is $1 / 166$ and $1 / 180$, depending on the attached functional group. Thus, in our model structures, we defined the length of the repeat unit substituents that have been attached periodically to a top $\mathrm{C}$ atom of the nanotube. Following previous work, ${ }^{44}$ the Fermi level is $21.3 \AA$ for the SWCNTs $(9,0)$ and $a=12.78 \AA$ for the SWCNTs featuring a single substituent oriented along the $z$-axis to match the experimental functionalization coverage, namely $a=(15,0)$. The resulting structures lead to a ratio of $1 / 180$ for both families. Fig. 1 shows the model functionalized structures. The substituents have been attached periodically to a top $\mathrm{C}$ atom of the nanotube. Following previous works, ${ }^{43}$ the Fermi level of the SWCNT has been calculated as the Dirac energy, which is defined at the center of the small energy gap.

\section{Photoelectrochemistry}

The photoelectrochemical experiments were performed using an AUTOLAB PGSTAT302N (Ecochemie) potentiostat. CV was carried out under a flow of nitrogen in a three-electrode cell using 0.1 $\mathrm{M} \mathrm{LiClO}_{4}$ (Sigma Aldrich) in dry acetonitrile as the supporting electrolyte. Graphite and $\mathrm{Ag} / \mathrm{AgCl}$ were used as the counter electrode and the reference electrode, respectively. The working electrode was prepared by spray coating $1 \mathrm{~mL}$ of SWCNT dispersions (0.02 $\left.\mathrm{mg} \mathrm{mL}^{-1}\right)$ on commercial FTO substrates (Solems S.A., France) covering an area of $c a .1 \mathrm{~cm}^{2}$. Samples were illuminated through a quartz window using a $150 \mathrm{~W}$ Xe arc lamp (300 $\mathrm{mW} \mathrm{cm}^{-2}$, LOT-Oriel 1.5 AM solar simulator, $\mathrm{GmbH}$, Germany). On-off $\mathrm{CV}$ at a scan rate of $0.002 \mathrm{~V} \mathrm{~s}^{-1}$ was carried out by intermittently interrupting the passage of the light beam with a frequency of about $0.03 \mathrm{~Hz}$. 


\section{Results and discussion}

\section{Functionalization of SWCNTs}

The functionalization degree on SWCNTs was first assessed by TGA (Fig. 2a). The organic moieties were quantified as follows:

$$
\mu \mathrm{mol} \mathrm{g}^{-1}=\frac{L(\%) \cdot 10^{4}}{M_{\mathrm{W}}\left(\mathrm{g} \mathrm{mol}^{-1}\right)},
$$

where $L$ corresponds to the weight loss observed at $450{ }^{\circ} \mathrm{C}$ (in \%) and the molar mass $\left(M_{\mathrm{W}}\right)$ is set for the expected desorbed moiety. The conversion factor $\left(10^{4}\right)$ provides data in units of $\mu \mathrm{mol} \mathrm{g}{ }^{-1} .^{34}$

In addition, we used another formula to estimate the functionalization degree in terms of the number of functional groups per $X$ carbon atoms in SWCNTs $(1 / X)$ :

$$
\frac{1}{X}=\frac{\mathrm{WL}(\%) \cdot 12\left(\mathrm{~g} \mathrm{~mol}^{-1}\right)}{R(\%) \cdot M_{\mathrm{W}}\left(\mathrm{g} \mathrm{mol}^{-1}\right)},
$$

where $X$ stands for the number of carbon atoms in the SWCNT per covalent functional group, WL (\%) is the weight loss in the range of $100-650{ }^{\circ} \mathrm{C}, R(\%)$ is the residual mass at $450{ }^{\circ} \mathrm{C}$ in the TGA plot and $M_{\mathrm{W}}$ is the molar mass of the desorbed moieties. ${ }^{34}$ This calculation considers SWCNT samples to be entirely constituted by carbon, which is indeed an approximation. The results obtained are summarized in Table 1 . The main conclusion extracted from the TGA is that both functional groups (benzenesulfonic acid or benzyl alcohol moieties) cover to a similar extent the SWCNT sidewalls. Having such a close degree of functionalization, further discussions regarding the observed differences between both functionalized SWCNTs (vide infra) should not be ascribed to a dissimilar functional group coverage.

The functionalization reactions may strongly affect some of the SWCNTs' optoelectronic properties, such as their UV/vis spectrum (Fig. S3 in the ESI $\dagger$ ). In fact, the diazonium reactions of SWCNTs with 4-aminobenzenesulfonic acid and with benzyl alcohol derivatives, resulting in A-SWCNTs and B-SWCNTs, respectively, cause a total bleaching of the absorption bands of SWCNTs.

\section{Raman spectroscopy}

Raman spectroscopy under ambient conditions was performed for a preliminary assessment of the effects of covalent functionalization
Table 1 Functionalization degree calculated from TGA

\begin{tabular}{lll}
\hline & A-SWCNTs & B-SWCNTs \\
\hline$\mu \mathrm{mol} \mathrm{g}^{-1}$ & 407 & 457 \\
$1 / X$ & 184 & 166
\end{tabular}

properties in A-SWCNTs and B-SWCNTs compared with SWCNTs (see Fig. 2b and c).

The RBM, in which all carbon atoms of the SWCNT vibrate radially in phase, is observed at low Raman shifts between 100 and $350 \mathrm{~cm}^{-1}$. The energy of the RBM depends inversely on the SWCNT diameter. ${ }^{25}$ In Fig. $2 b$ and $c$ inset, changes in the $\mathrm{RBM}$ intensity and frequency are observed due to chemical functionalization. All the RBM bands of functionalized SWCNTs showed either bleaching or amplification compared with SWCNTs. However, nanotubes with mainly metallic character present large modifications in the $\mathrm{RBM}$ intensity ( $\mathrm{E}_{11}^{\mathrm{M}}$ yellow region) upon functionalization, in good agreement with the previous literature. ${ }^{9,34}$

The tangential modes are the most intense in SWCNTs and form the G-band, which is related to in-plane $\mathrm{C}-\mathrm{C}$ bond stretching, at around $1590 \mathrm{~cm}^{-1} \cdot{ }^{25}$ The line shape of the G-band provides additional information about the metallic and semiconducting SWCNTs in resonance. With the $2.33 \mathrm{eV}$ laser line, the G-band is highly asymmetric, which indicates that the band is narrower, indicating that mostly semiconducting SWCNTs are in resonance. ${ }^{45}$ The intensity and the position of the G-band change with functionalization (see Table 2). This could indicate changes in the electronic structure, in doping or in the resonance conditions of SWCNTs contributing to the G-band. This result suggested that the functional groups attached to the SWCNTs lead to a change in the doping level of SWCNTs. ${ }^{28}$ In addition, the SWCNT spectra presented in Fig. 2 reveal information about structural defects. The disorder-induced mode (the D-band) is observed at 1329 and $1312 \mathrm{~cm}^{-1}$ using 2.33 and $1.96 \mathrm{eV}$ excitation energies, respectively. ${ }^{25}$ The D-band intensity depends on the laser energy and also correlates with the degree of functionalization. ${ }^{34,46}$ The D-band increases with functionalization due to the formation of $\mathrm{sp}^{3}$ defects on the nanotube surface where the functional group is covalently attached to the SWCNT wall (Table 2).
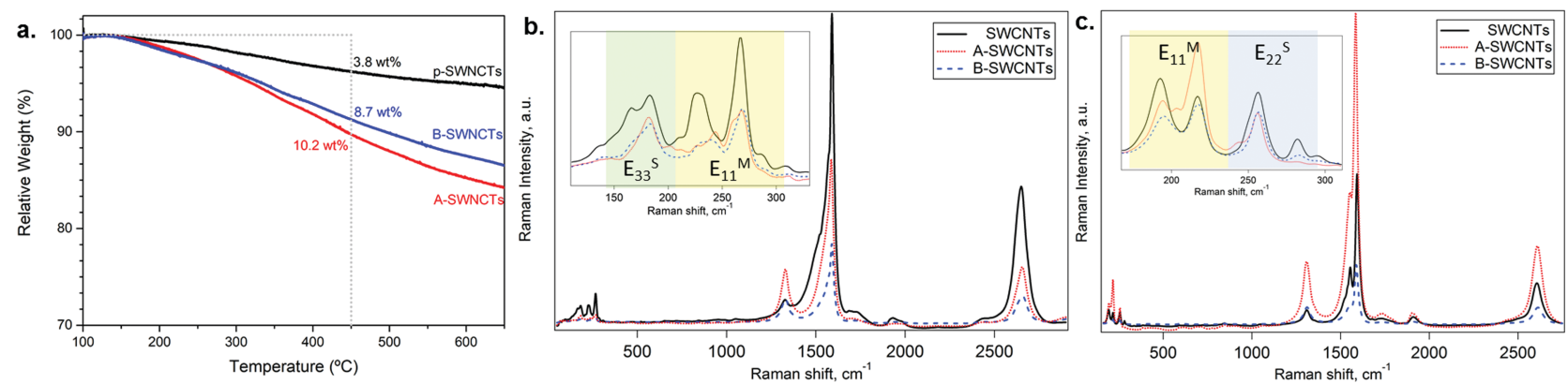

Fig. 2 (a) TGA in an $\mathrm{N}_{2}$ atmosphere for SWCNTs (black lines), A-SWCNTs (red line) and B-SWCNTs (blue lines) in the range of $100-650{ }^{\circ} \mathrm{C}$. Dotted lines delimit the range in which weight losses are considered for calculations. (b and c) Resonance Raman spectra and the RBM region (inset) of SWCNTs (black lines), A-SWCNTs (red dotted lines), and B-SWCNTs (blue dashed lines) excited by different laser lines: (b) $2.33 \mathrm{eV}$ and (c) $1.96 \mathrm{eV}$. In the RBM region (inset), the green, yellow and blue regions correspond to the $E_{33}^{S}, E_{11}^{M}$ and $E_{22}^{S}$ electronic transitions, respectively. Each spectrum is an average based on $49(2.33 \mathrm{eV})$ and 900 spectra $(1.96 \mathrm{eV})$ measured at different points. 
Table 2 G-band shift and the $I_{D} / I_{G}$ intensity ratio of the SWCNTs, A-SWCNTs and B-SWCNTS

\begin{tabular}{llllll}
\hline & $2.33 \mathrm{eV}$ & & & $1.96 \mathrm{eV}$ & \\
\cline { 2 - 3 } & G-Band shift $\left(\mathrm{cm}^{-1}\right)$ & $I_{\mathrm{D}} / I_{\mathrm{G}}$ & & G-Band shift $\left(\mathrm{cm}^{-1}\right)$ & $I_{\mathrm{D}} / I_{\mathrm{G}}$ \\
\hline SWCNTs & 1593 & 0.07 & & 1593 & 0.09 \\
A-SWCNTs & 1585 & 0.32 & & 1585 & 0.20 \\
B-SWCNTs & 1590 & 0.29 & 1588 & 0.28
\end{tabular}

\section{In situ Raman spectroelectrochemistry}

In situ Raman spectroelectrochemistry was applied to investigate in detail the changes induced in the electronic structure of SWCNTs upon functionalization. Electrochemical charging can be understood using a double layer capacitor model, where the SWCNTs act as the working electrode and the charge carriers are injected into the SWCNTs, while the electrolyte ions compensate the charge. Extra electrons or holes from the circuit are inserted into the SWCNTs, causing changes in the electronic structure and these changes can be followed by Raman spectroscopy. Consequently, when the potential is changing, the charge transfer leads to a shift in the Fermi level. Taking into account the fact that the RBM intensity of SWCNTs is sensitive to the doping level, ${ }^{47,48}$ we have here specifically followed the changes upon functionalization with the electron-withdrawing and electron-donating molecules.

The potential-dependent Raman spectra for the RBM region of the SWCNTs in the acetonitrile electrolyte solution are shown, as an example, in Fig. 3 (the spectra of A- and B-SWCNTs are shown in Fig. S4 and S5 in the ESI $\dagger$ ). Sections (a) and (b) show the spectra obtained using 2.33 and $1.96 \mathrm{eV}$ laser excitation energies, respectively. The positions of the most intense RBM bands are

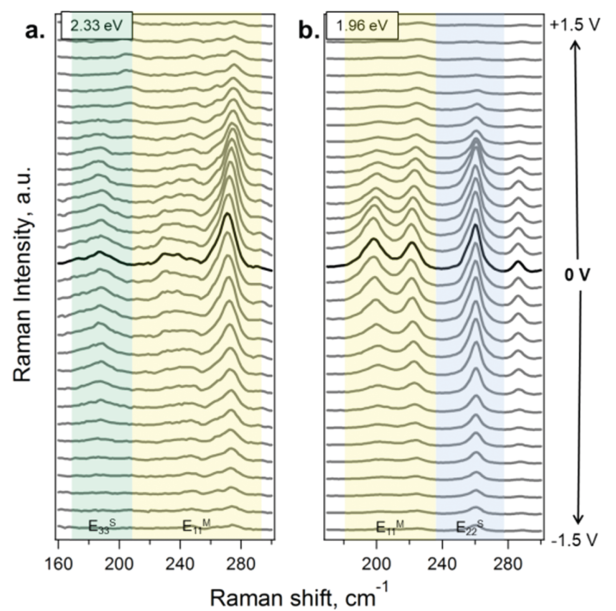

Fig. 3 Raman spectra of the SWCNTs at different potentials in the RBM spectral region. The electrode potential range is from -1.5 to $1.5 \mathrm{~V} v \mathrm{v}$. the $\mathrm{Ag}$ pseudoreference electrode (from bottom to top). The spectra in bold correspond to the $0 \mathrm{~V}$ potential. The electrochemical potential change between the spectra is $0.1 \mathrm{~V}$. The spectra were acquired using (a) 2.33 and (b) $1.96 \mathrm{eV}$ laser excitation energies. The green, yellow and blue parts correspond to the regions where the used laser excitation energy matches $E_{33}^{S}, E_{11}^{M}$ and $E_{22}^{S}$ electronic transitions in the probed nanotubes, respectively. The spectra are offset for clarity. studied and the diameters of SWCNTs in resonance are estimated: ${ }^{49}$ $183\left(d_{\mathrm{t}}=1.33 \mathrm{~nm}\right), 268 \mathrm{~cm}^{-1}\left(d_{\mathrm{t}}=0.89 \mathrm{~nm}\right)$ in the case of laser energy $E_{\text {laser }}=2.33 \mathrm{eV}$, and $193\left(d_{\mathrm{t}}=1.24 \mathrm{~nm}\right)$ and $257 \mathrm{~cm}^{-1}$ $\left(d_{\mathrm{t}}=0.93 \mathrm{~nm}\right)$ at $1.96 \mathrm{eV}$ laser excitation energy. When applying positive potentials, from $0 \mathrm{~V}$ to $+1.5 \mathrm{~V}$, in steps of $0.1 \mathrm{~V}$, the Fermi level $\left(E_{\mathrm{F}}\right)$ is downshifted (introducing holes into the $\pi$-band). The opposite occurs when negative potentials are applied, from $0 \mathrm{~V}$ to $-1.5 \mathrm{~V}$; the electron density increases due to the upshift in the $E_{\mathrm{F}}$ value (introducing electrons into the $\pi$-band). In this way, when the $E_{\mathrm{F}}$ value reaches a van Hove singularity (vHs), the corresponding electronic transition is blocked, and the Raman signal is bleached.

For a more detailed analysis of the SWCNT metallic/semiconducting character and the doping level, the dependence of the RBM intensity on the electrode potential at the selected laser lines was studied (see Fig. S6 in the ESI†). A difference in the profiles of the Raman intensity $\left(I_{\mathrm{RBM}}\right) v s$. applied potential $\left(E_{\text {app }}\right)$ of metallic and semiconducting SWCNTs has been previously reported. ${ }^{47,48}$ For semiconducting SWCNTs in resonance at $2.33 \mathrm{eV}$, the profile $I_{\mathrm{RBM}} v s$. $E_{\mathrm{app}}$ exhibits a plateau close to $0 \mathrm{~V}$. The $I_{\mathrm{RBM}}$ profile is not significantly attenuated close to $0 \mathrm{~V}$ and starts changing only after the electrode potential reaches the first vHs. In contrast, the intensity of the band for metallic SWCNTs is attenuated at potentials close to $0 \mathrm{~V}$. A similar effect occurs for the SWCNT in resonance at the laser excitation energy of $1.96 \mathrm{eV}$. Therefore, for semiconducting SWCNTs, the profile of $I_{\mathrm{RBM}}$ vs. $E_{\text {app }}$ at around $0 \mathrm{~V}$ is a plateau and for the metallic SWCNT, it is sharp and shows a maximum. So one can distinguish between metallic and semiconducting SWCNTs. ${ }^{47}$ These assignments obtained using the $I_{\mathrm{RBM}} v s$. $E_{\text {app }}$ profiles are in agreement with the electronic transitions in the Kataura plot. ${ }^{49-51}$

The $I_{\mathrm{RBM}} v s$. $E_{\mathrm{app}}$ profiles of the SWCNT, A- and B-SWCNT samples are shown in Fig. 4. The $I_{\mathrm{RBM}}$ profiles of the bands in resonance via the $\mathrm{E}_{11}^{\mathrm{M}}$ transition reach a maximum at a given optimum potential. We found that maximum $I_{\mathrm{RBM}}$ for the metallic SWCNT vs. $E_{\text {app }}$ is shifted for the functionalized samples in comparison with SWCNTs (Fig. 4b and c). In the case of semiconducting SWCNTs, there is no noticeable shift because of the occurrence of a plateau instead of a maximum. For both laser excitation energies, the profiles of $I_{\mathrm{RBM}}$ in resonance via $\mathrm{E}_{11}^{\mathrm{M}}$ are downshifted for A-SWCNTs and upshifted for B-SWCNTs, in comparison to SWCNTs. This indicates that doping is induced on the SWCNTs by the functional groups attached to their walls. The benzenesulfonic acid moiety has a large electron affinity due to its structure composed of a benzene ring attached in the para position to a sulfur(vI) atom in a central part of a $\mathrm{SO}_{3} \mathrm{H}$ group. With three electronegative oxygen atoms, the $\mathrm{SO}_{3} \mathrm{H}$ group is strongly electronwithdrawing. It is necessary to apply an additional negative potential to reach the maximum peak of Raman intensity, pointing out that the A-SWCNTs are p-doped and the $E_{\mathrm{F}}$ value is downshifted. In this way the valence band vHs is depleted and it is necessary to apply an extra negative potential to reach the neutral point. On the other hand, in the case of B-SWCNTs the maximum peak is shifted to positive potentials. This means that the $\mathrm{E}_{11}^{\mathrm{M}}$ transition becomes occupied (n-doped, filling) because $E_{\mathrm{F}}$ is upshifted, as benzyl alcohol gives electrons to the SWCNT electronic structure. 


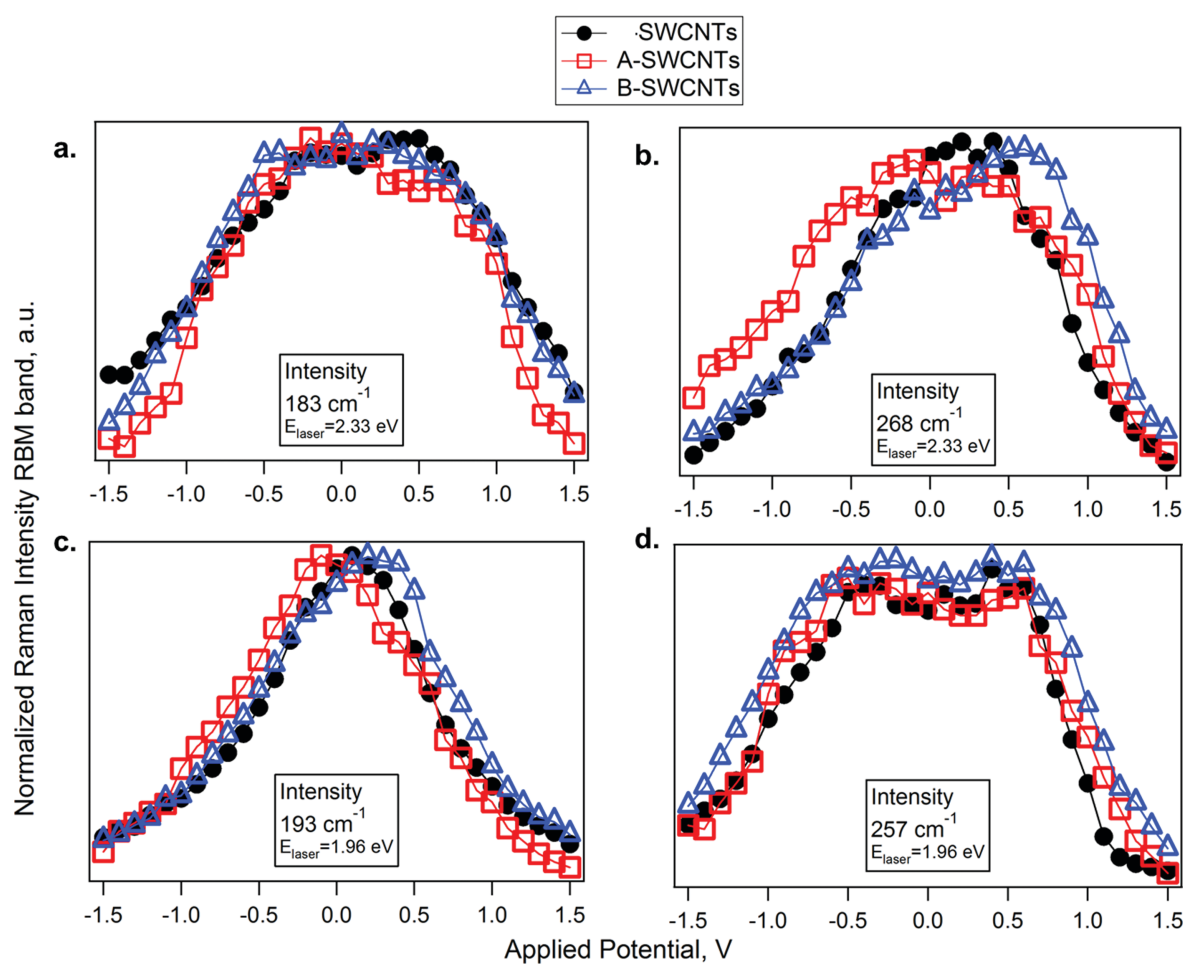

Fig. $4 I_{\text {RBM }}$ Vs. $E_{\text {app }}$ for SWCNTs (black circles), A-SWCNTs (empty red squares) and B-SWCNTs (empty blue triangles) in resonance at $2.33 \mathrm{eV}$. (a and b) and $1.96 \mathrm{eV}$ (c and d) for: (a) the band at $183 \mathrm{~cm}^{-1}$ (semiconducting $E_{33}^{S}$ ), (b) the band at $268 \mathrm{~cm}^{-1}$ (metallic $\mathrm{E}_{11}^{\mathrm{M}}$ ), (c) the band at $193 \mathrm{~cm}^{-1}$ (metallic $\mathrm{E}_{11}^{\mathrm{M}}$ ), and (d) the band at $257 \mathrm{~cm}^{-1}$ (semiconducting $\mathrm{E}_{22}^{\mathrm{S}}$ ).

\section{Theoretical calculations}

DFT calculations have been carried out to support the Raman spectroelectrochemistry results. As an example, we study two SWCNT families in resonance with the laser lines at 2.33 and $1.96 \mathrm{eV}$ (Fig. 5). The densities of states (DOS) of the functionalized SWCNTs have similar shapes but show shift in energy due to their different donor/acceptor substituents.

Theoretical calculations support that there is a shift in the Fermi level and the vHs due to the functionalization. As observed experimentally, the A-SWCNT functionalization yields a downward shift of the SWCNT Fermi level. On the other hand, the B-SWCNT functionalization takes place at the origin of an

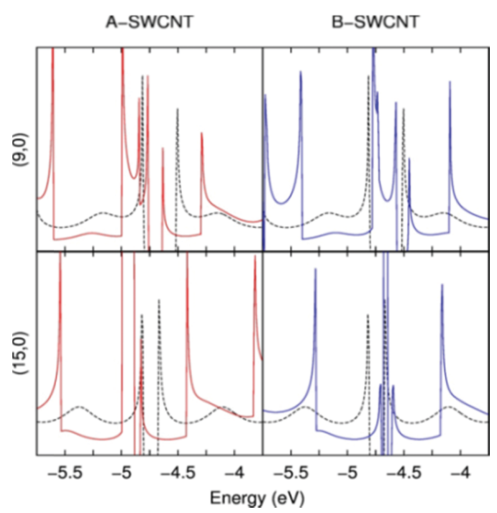

Fig. 5 DOS of the SWCNTs (dashed lines), A-SWCNTs (red lines) and B-SWCNTs (blue lines) for the two families studied.
Table 3 Absolute values of the Fermi level of the studied SWCNTs in eV. As mentioned in the Methods section, this value has been taken as the Dirac energy

\begin{tabular}{llll}
\hline$E_{\mathrm{F}}(\mathrm{eV})$ & SWCNTs & A-SWCNTs & B-SWCNTs \\
\hline$(9,0)$ & -4.65 & -4.69 & -4.51 \\
$(15,0)$ & -4.73 & -4.85 & -4.62
\end{tabular}

upward shift of the Fermi level with respect to the SWCNTs. The calculated shifts of the Fermi level are given in Table 3.

\section{Photoelectrochemistry}

The unique densities of states of SWCNTs allow the electronhole separation upon photocurrent generation when they are exposed to light. ${ }^{52,53}$ Therefore, it can be expected that the doping effect induced in the functionalized SWCNTs would also be reflected in the photoelectrochemical properties. Testing the n-/p-doped functionalized SWCNT films in a three-electrode electrochemical cell, we study here the changes that occur in the photoresponse when chemical doping is introduced in the electronic structure of SWCNTs.

Dark voltammograms for SWCNT, A-SWCNT and B-SWCNT film electrodes show the features that have been previously observed in the literature for similar systems, i.e. a 'bow tie' shape ${ }^{54,55}$ with the narrowest part indicating the position of the Fermi level (see Fig. S7 in the ESI $\dagger$ ).

Fig. 6 summarizes the changes observed by CV under illumination. In particular, the current in the cathodic branch 

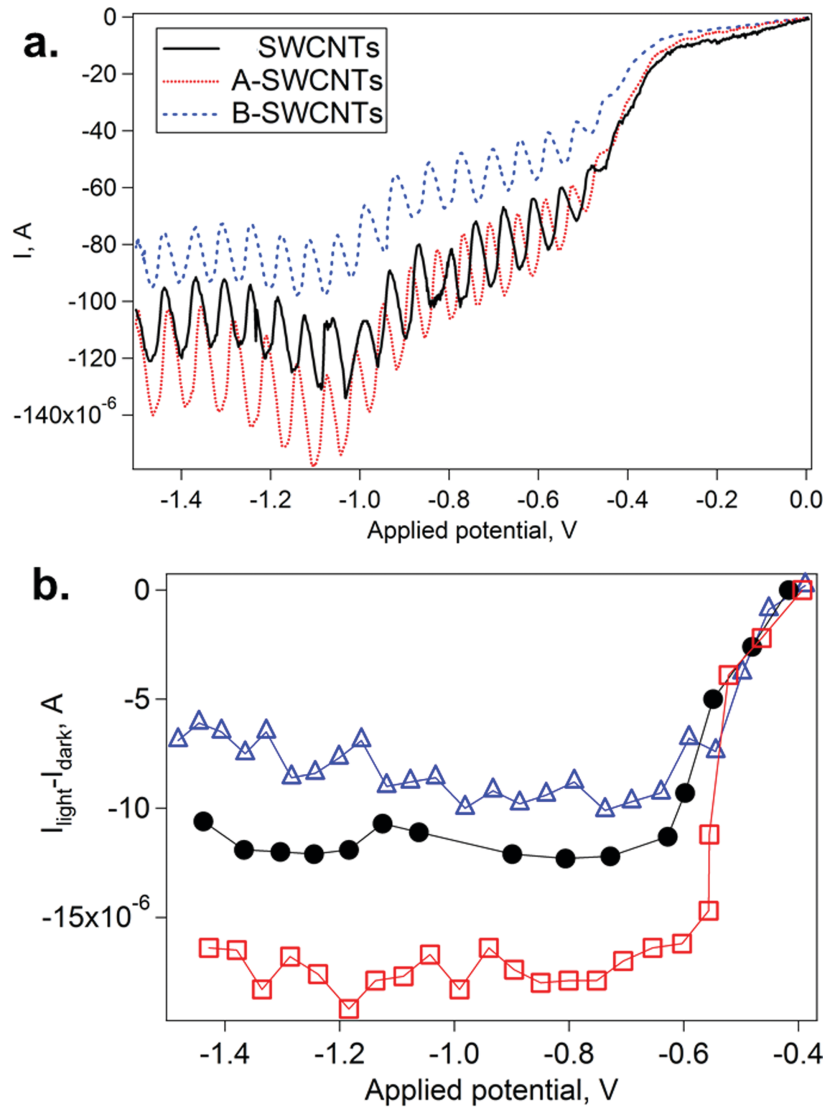

Fig. 6 (a) Light-dark cathodic scans for SWCNT (black lines), A-SWCNT (red dotted lines) and B-SWCNT (blue dashed lines) films in $0.1 \mathrm{M} \mathrm{LiClO}_{4}$ acetonitrile. Scan rates of $2 \mathrm{mV} \mathrm{s}^{-1}$. (b) Photocurrent ( / light $-I_{\text {dark }}$ ) as a function of the $E_{\text {app }}$ value for SWCNT (black circles), A-SWCNT (empty red squares) and B-SWCNT (empty blue triangles) films.

of the scan is modified when the lamp is alternately switched on and off (Fig. 6a). Thus, the photocurrent can be calculated as the difference between consecutive on and off currents $\left(I_{\text {light }}-I_{\text {dark }}\right)$ at various $E_{\text {app }}$ values (Fig. 6b). Photoanodic activity, typically less than $1 \mu \mathrm{A}$, was much lower than the photocathodic one (see Fig. S8, ESI $\dagger$ ). When the three SWCNT electrodes are irradiated, a negative photocurrent appears at potentials lower than $-0.4 \mathrm{~V}$. In previous literature, photoelectroactivity for SWCNTs has been observed in another electrochemical reaction. ${ }^{56}$ In our work, the photocathodic process has to be observed at low scan rates $\left(2 \mathrm{mV} \mathrm{s}^{-1}\right)$, and the values observed for the photocurrent reached the noticeable value of $c a .20 \mu \mathrm{A}$. In these cases, the effects of the SWCNT functionalization are observed: A-SWCNTs show higher photocathodic currents than SWCNTs, indicating p-doping, while the contrary effect is observed for B-SWCNTs, indicating n-doping. Differences in photoanodic current between SWCNT, A-SWCNT and B-SWCNT films are not significant (see Fig. S9, ESI $\dagger$ ). However, in the case of photocathodic current, the differences between SWCNT, A-SWCNT and B-SWCNT films are above $5 \mu \mathrm{A}$. These results are fully consistent with those described in the preceding sections, and indicate that the photoelectrochemical properties of SWCNTs can be tuned by chemical functionalization.
Moreover, the chemically modified photoresponse can be predicted by using spectroelectrochemical techniques in a reliable manner, opening new possibilities in fields such as solar energy conversion and photosensors.

\section{Conclusions}

In summary, we have been able to determine the type of doping (n-/p-) of a bulk sample of chemically functionalized HiPco SWCNTs using an in situ Raman spectroelectrochemistry setup, studying the development of the RBM intensities in resonance via $\mathrm{E}_{11}^{\mathrm{M}}$ during electrochemical charging. This method, which is validated by DFT calculations, can constitute a routine test to estimate the electronic effects of SWCNT functionalization. It can also be used to predict the electronic properties of functionalized SWCNTs in devices. As a proof of principle, functionalized SWCNT films were used as electrodes in photoelectrochemistry measurements. The current under light irradiation was influenced by the functional group, in agreement with the spectroelectrochemical information and theoretical calculations. The procedure can be extended to other functional groups and/or other reactions in the chemistry of SWCNTs.

\section{Conflicts of interest}

There are no conflicts to declare.

\section{Acknowledgements}

The authors acknowledge financial support from the European Union FP7 RADDEL programme (ITN Marie Curie Actions PEOPLE-2011-290023) and the MEYS Inter Excellence Project no. LTC18039 and H2020-MSCA RISE Agreement no. 734834 (INFUSION). This work at the Instituto de Carboquímica has been funded by the MINECO and the European Regional Development Fund (ENE 2016-79282-C5-1-R), the Government of Aragon (T03-17R), and the European Commission (H2020MSCA-ITN-2014-ETN 642742 'Enabling Excellence'). The work in Mons is supported by the European Union H2020 iSwitch programme (H2020-MSCA-ITN-2014-ETN 642196). Computational resources in Mons were provided by the Consortium des Équipements de Calcul Intensif (CÉCI) funded by the Belgian National Fund for Scientific Research (F.R.S.-FNRS) under Grant 2.5020.11. J. C. is an FNRS research director. The authors acknowledge the assistance provided by the Research Infrastructures NanoEnviCz (Project No. LM2015073) supported by the Ministry of Education, Youth and Sports of the Czech Republic and the project Pro-NanoEnviCz (Reg. no. CZ.02.1.01/ 0.0/0.0/16_013/0001821) supported by the Ministry of Education, Youth and Sports of the Czech Republic and the European Union European Structural and Investments Funds in the frame of Operational Programme Research Development and Education. J. M. G.-D. is grateful to Spanish Ministry of Science, Innovation and Universities for his 'Juan de la Cierva - Incorporación' 
research grant. A. S. is grateful to Dr Sara Costa and Dr Johan Ek Weis for fruitful discussions and helpful comments.

\section{Notes and references}

1 V. Georgakilas, J. A. Perman, J. Tucek and R. Zboril, Chem. Rev., 2015, 115, 4744-4822.

2 M. F. L. De Volder, S. H. Tawfick, R. H. Baughman and A. J. Hart, Science, 2013, 339, 535-539.

3 K. Chen, W. Gao, S. Emaminejad, D. Kiriya, H. Ota, H. Y. Yin Nyein, K. Takei and A. Javey, Adv. Mater., 2016, 28, 4397-4414.

4 L. Yu, C. Shearer and J. Shapter, Chem. Rev., 2016, 116, 13413-13453.

5 P. Prakash, K. Mohana Sundaram and M. Anto Bennet, Renewable Sustainable Energy Rev., 2018, 89, 194-203.

6 S. A. Hodge, M. K. Bayazit, K. S. Coleman and M. S. P. Shaffer, Chem. Soc. Rev., 2012, 41, 4409-4429.

7 S. Mallakpour and S. Soltanian, RSC Adv., 2016, 6, 109916. 8 A. Setaro, J. Phys.: Condens. Matter, 2017, 29, 423003.

9 M. S. Strano, C. A. Dyke, M. L. Usrey, P. W. Barone, M. J. Allen, H. Shan, C. Kittrell, R. H. Hauge, J. M. Tour and R. E. Smalley, Science, 2003, 301, 1519-1522.

10 V. Sgobba and D. M. Guldi, Chem. Soc. Rev., 2009, 38, 165-184. 11 K. Dirian, M. A. Herranz, G. Katsukis, J. Malig, L. RodriguezPerez, C. Romero-Nieto, V. Strauss, N. Martin and D. M. Guldi, Chem. Sci., 2013, 4, 4335-4353.

12 M. Scardamaglia, C. Struzzi, F. J. Aparicio Rebollo, P. De Marco, P. R. Mudimela, J.-F. Colomer, M. Amati, L. Gregoratti, L. Petaccia, R. Snyders and C. Bittencourt, Carbon, 2015, 83, 118-127.

13 J. L. Bahr and J. M. Tour, Chem. Mater., 2001, 13, 3823-3824.

14 G. Schmidt, S. Gallon, S. Esnouf, J.-P. Bourgoin and P. Chenevier, Chem. - Eur. J., 2009, 15, 2101-2110.

15 B. Dinesh, A. Bianco and C. Menard-Moyon, Nanoscale, 2016, 8, 18596-18611.

16 Y. Piao, B. Meany, L. R. Powell, N. Valley, H. Kwon, G. C. Schatz and Y. Wang, Nat. Chem., 2013, 5, 840.

17 M. Kim, L. Adamska, N. F. Hartmann, H. Kwon, J. Liu, K. A. Velizhanin, Y. Piao, L. R. Powell, B. Meany, S. K. Doorn, S. Tretiak and Y. Wang, J. Phys. Chem. C, 2016, 120, 11268-11276.

18 L. R. Powell, Y. Piao and Y. Wang, J. Phys. Chem. Lett., 2016, 7, 3690-3694.

19 A. López-Bezanilla, F. Triozon, S. Latil, X. Blase and S. Roche, Nano Lett., 2009, 9, 940-944.

20 F. Teichert, A. Zienert, J. Schuster and M. Schreiber, Comput. Mater. Sci., 2017, 138, 49-57.

21 J. L. Blackburn, A. J. Ferguson, C. Cho and J. C. Grunlan, Adv. Mater., 2018, 30, 1704386.

22 T. Ketolainen, V. Havu, E. Ö. Jónsson and M. J. Puska, Phys. Rev. Appl., 2018, 9, 034010.

23 C. Romero-Nieto, R. García, M. Á. Herranz, L. RodríguezPérez, M. Sánchez-Navarro, J. Rojo, N. Martín and D. M. Guldi, Angew. Chem., Int. Ed., 2013, 52, 10216-10220.

24 N. V. Kurnosov, V. S. Leontiev, A. S. Linnik and V. A. Karachevtsev, Chem. Phys. Lett., 2015, 623, 51-54.
25 M. S. Jorio, R. Saito and G. Dresselhaus, Raman Spectroscopy in Graphene Related Systems, J. W. Sons, 2011.

26 B. Nikolić, I. Milošević and M. Damnjanović, J. Phys. Chem. C, 2014, 118, 20576-20584.

27 H.-J. Shin, S. M. Kim, S.-M. Yoon, A. Benayad, K. K. Kim, S. J. Kim, H. K. Park, J.-Y. Choi and Y. H. Lee, J. Am. Chem. Soc., 2008, 130, 2062-2066.

28 R. Voggu, C. S. Rout, A. D. Franklin, T. S. Fisher and C. N. R. Rao, J. Phys. Chem. C, 2008, 112, 13053-13056.

29 C. N. R. Rao and R. Voggu, Mater. Today, 2010, 13, 34-40.

30 K. Ladislav and D. Lothar, ChemPhysChem, 2007, 8, 974-998.

31 D. Ibañez, J. Garoz-Ruiz, D. Plana, A. Heras, D. J. Fermín and A. Colina, Electrochim. Acta, 2016, 217, 262-268.

32 M. Kierkowicz, E. Pach, A. Santidrián, E. Tobías-Rossell, M. Kalbáč, B. Ballesteros and G. Tobias, ChemNanoMat, 2016, 2, 108-116.

33 Y. Zhai, Z. Zhu, S. Zhou, C. Zhu and S. Dong, Nanoscale, 2018, 10, 3089-3111.

34 J. M. González-Domínguez, A. Santidrián, A. Criado, C. Hadad, M. Kalbáč and T. D. Ros, Chem. - Eur. J., 2015, 21, 18631-18641.

35 V. V. Pavlishchuk and A. W. Addison, Inorg. Chim. Acta, 2000, 298, 97-102.

36 W. Kohn, Rev. Mod. Phys., 1999, 71, 1253-1266.

37 D. Sánchez-Portal, P. Ordejón, E. Artacho and J. M. Soler, Int. J. Quantum Chem., 1997, 65, 453-461.

38 J. P. Perdew, K. Burke and M. Ernzerhof, Phys. Rev. Lett., 1996, 77, 3865-3868.

39 J. M. Soler, E. Artacho, J. D. Gale, A. García, J. Junquera, P. Ordejón and D. Sánchez-Portal, J. Phys.: Condens. Matter, 2002, 14, 2745.

40 N. Troullier and J. Martins, Solid State Commun., 1990, 74, 613-616.

41 R. Dovesi, R. Orlando, B. Civalleri, C. Roetti, R. Saunders Victor and M. Zicovich-Wilson Claudio, Z. Kristallogr. Cryst. Mater., 2005, 220, 571.

42 C. Adamo and V. Barone, J. Chem. Phys., 1999, 110, 6158-6170. 43 H. C. Bai and L. You, Int. J. Sci., 2014, 1, 48-52.

44 L.-C. Yin, H.-M. Cheng, R. Saito and M. S. Dresselhaus, Carbon, 2011, 49, 4774-4780.

45 M. A. Pimenta, A. Marucci, S. A. Empedocles, M. G. Bawendi, E. B. Hanlon, A. M. Rao, P. C. Eklund, R. E. Smalley, G. Dresselhaus and M. S. Dresselhaus, Phys. Rev. B: Condens. Matter Mater. Phys., 1998, 58, R16016.

46 J. Maultzsch, S. Reich and C. Thomsen, Phys. Rev. B: Condens. Matter Mater. Phys., 2001, 64, 121407.

47 M. Kalbac, H. Farhat, L. Kavan, J. Kong, K.-i. Sasaki, R. Saito and M. S. Dresselhaus, ACS Nano, 2009, 3, 2320-2328.

48 F. Dragin, A. Pénicaud, M. Iurlo, M. Marcaccio, F. Paolucci, E. Anglaret and R. Martel, ACS Nano, 2011, 5, 9892-9897.

49 P. T. Araujo, P. B. C. Pesce, M. S. Dresselhaus, K. Sato, R. Saito and A. Jorio, Phys. E, 2010, 42, 1251-1261.

50 H. Kataura, Y. Kumazawa, Y. Maniwa, I. Umezu, S. Suzuki, Y. Ohtsuka and Y. Achiba, Synth. Met., 1999, 103, 2555-2558. 
51 D. Zhang, J. Yang, F. Yang, R. Li, M. Li, D. Ji and Y. Li, Nanoscale, 2015, 7, 10719-10727.

52 S. Barazzouk, S. Hotchandani, K. Vinodgopal and P. V. Kamat, J. Phys. Chem. B, 2004, 108, 17015-17018.

53 M. Engel, M. Steiner, R. S. Sundaram, R. Krupke, A. A. Green, M. C. Hersam and P. Avouris, ACS Nano, 2012, 6, 7303-7310.
54 S. A. Hodge, M. K. Bayazit, H. H. Tay and M. S. P. Shaffer, Nat. Commun., 2013, 4, 1989.

55 S. A. Hodge, S. Fogden, C. A. Howard, N. T. Skipper and M. S. P. Shaffer, ACS Nano, 2013, 7, 1769-1778.

56 M. A. Bissett and J. G. Shapter, J. Electrochem. Soc., 2011, 158, K53-K57. 